Revue d'histoire de l'Amérique française

REVUE D.HISTOIRE DE L'AMÉRIQUE FRANÇAISE

\title{
HANAWAY, Joseph et Richard CRUESS, McGill Medicine, 1: The First Half Century, 1829-1885 (Montréal, McGill-Queen's University Press, 1996), 219 p.
}

\section{Denis Goulet}

Volume 50, numéro 4, printemps 1997

URI : https://id.erudit.org/iderudit/305606ar

DOI : https://doi.org/10.7202/305606ar

Aller au sommaire du numéro

Éditeur(s)

Institut d'histoire de l'Amérique française

ISSN

0035-2357 (imprimé)

1492-1383 (numérique)

Découvrir la revue

Citer ce compte rendu

Goulet, D. (1997). Compte rendu de [HANAWAY, Joseph et Richard CRUESS, McGill Medicine, 1: The First Half Century, 1829-1885 (Montréal, McGill-Queen's University Press, 1996), 219 p.] Revue d'histoire de l'Amérique française, 50(4),

619-621. https://doi.org/10.7202/305606ar d'utilisation que vous pouvez consulter en ligne.

https://apropos.erudit.org/fr/usagers/politique-dutilisation/ 
HANAWAY, Joseph et Richard CRUESS, McGill Medicine, 1: The First Half Century, 1829-1885 (Montréal, McGill-Queen's University Press, 1996), $219 \mathrm{p}$.

L'histoire des institutions médicales au Québec connaît depuis quelques années un essor important avec la parution d'un certain nombre de travaux qui rendent compte de l'évolution des institutions hospitalières, des structures de santé publique et des facultés de médecine. Cependant, l'ouvrage d'Hanaway et Cruess comble certainement une lacune importante de l'historiographie puisqu'aucune monographie n'avait jusqu'à ce jour abordé spécifiquement l'histoire de la première faculté de médecine universitaire en territoire canadien. Il s'agit ici du volume 1 (1829-1885) couvrant la période de fondation et de consolidation de cette importante faculté. L'ouvrage, facile d'accès aux non-spécialistes, est par ailleurs modeste puisque le texte principal ne compte qu'une centaine de pages, largement illustrées de gravures et de photographies, le reste étant constitué de notices biographiques, de listes d'étudiants gradués, etc. L'absence de bibliographie, omission qui sera probablement réparée dans le tome 2, est plutôt agaçante.

Privilégiant une approche descriptive et chronologique, les auteurs Hanaway et Cruess, respectivement professeur de neurologie et doyen retraité de l'université McGill, présentent avec précision les origines et le développement d'une institution d'enseignement dont la réputation débordera largement au tournant du $\mathrm{XX}^{\mathrm{e}}$ siècle les frontières canadiennes. On peut cependant s'interroger sur la pertinence de la période indiquée dans le titre de l'ouvrage (1829-1885) puisque la faculté de médecine de McGill, comme le montrent les auteurs, est directement issue de la Montreal Medical Institution mise sur pied en 1823. Du reste, il est clairement mentionné dans l'ouvrage que les quatre professeurs de la faculté en 1829 sont les quatre fondateurs de la Montreal Medical Institution. Les auteurs font reposer leur périodisation sur le critère de la création de l'université McGill en 1829. 
L'intérêt principal de cet ouvrage tient à ce que les auteurs ont réussi d'une part, à recréer, par des descriptions minutieuses et certaines anecdotes, le type d'enseignement qui prévalait à McGill et, d'autre part, à présenter la période de transition d'une formation médicale centrée sur les leçons magistrales vers une formation de plus en plus axée sur des travaux pratiques et des travaux anatomopathologiques, grâce, en particulier, à l'arrivée de William Osler. Par ailleurs, les auteurs ont assez fidèlement précisé les modèles d'enseignement adoptés par les membres de la faculté, modèles largement inspirés de la faculté de médecine d'Édimbourg jusqu'à la décennie 1870 . C'est, du reste, grâce aux contacts étroits établis entre les professeurs de McGill et les grandes facultés britanniques que seront introduites, relativement tôt en territoire canadien, les premières pratiques antiseptiques issues notamment du chirurgien anglais Joseph Lister.

Malgré des qualités indéniables, cet ouvrage comporte de sérieuses lacunes sur le plan méthodologique. Si les auteurs sont soucieux de situer les influences britanniques dans le développement de la faculté, en revanche, ils ne font aucun cas des relations directes ou indirectes qui se sont nouées entre les institutions médicales francophones et anglophones. Le choix des auteurs est évident: rendre compte des développements internes de la faculté, en négligeant le contexte externe. Ce manque d'intérêt pour tout ce qui touche le développement de la médecine francophone - la faculté de médecine de McGill n'est tout de même pas située en Australie - se reflète de façon fâcheuse dans l'historiographie utilisée. Qu'on ne fasse aucune mention de l'ouvrage de Boissonnault, Histoire de la faculté de médecine de Laval (1953), constitue un oubli certes important, mais que les auteurs ne fassent aucune mention de l'ouvrage publié en 1993, Histoire de la faculté de médecine de l'Université de Montréal 1843-1993, constitue un irritant majeur, et pas seulement parce que j'en suis l'auteur. Même l'ouvrage de Bernier, La médecine au Québec. Naissance et évolution d'une profession, ne fait l'objet d'aucune mention. Ce type d'ouvrage me rappelle celui de Sclater Lewis qui, plusieurs années auparavant, avait publié une histoire du Royal Victoria Hospital en ignorant totalement le contexte sociomédical québécois. Il me faut aussi mentionner les lacunes des auteurs en ce qui regarde les travaux récents sur l'histoire de la médecine canadienne. L'ouvrage de Travill, $M e$ dicine at Queen's 1854-1920, publié en 1988, n'est pas mentionné et même l'article de Connor publié en 1993 sur les débuts de l'antisepsie en territoire canadien est ignoré. Par contre, les auteurs se réfèrent fréquemment aux travaux d'Abbott (1931) et d'Heagerty (1928). L'ouvrage aurait aussi pu être plus étoffé. Par exemple, il n'est fait que brièvement mention de l'incorporation du Collège des médecins de la Province de Québec. Or, les membres de la faculté de médecine de McGill ont grandement contribué au débat entourant l'organisation de la profession dans le Bas-Canada. Je pourrais donner d'autres exemples de ce type. En somme, malgré une bonne description du développement interne de la faculté, cet ouvrage demeure dans l'en- 
semble décevant et s'inscrit dans la tradition des monographies orientées vers la promotion de l'institution analysée. 